\title{
The influence of depolarization field on dielectric and pyroelectric properties of ferroelectric films
}

\author{
M.D. Glinchuk, E.A. Eliseev, V.A. Stephanovich ${ }^{1)}$ \\ Institute for Problems of Materials Science, NAS of Ukraine, Krjijanovskogo 3, 03142 Kiev, Ukraine \\ Phone: +380 (44) 444-2131; e-mail glin@materials.kiev.ua \\ ${ }^{1)}$ Institute of Mathematics, Opole University, 45-052 Opole, Poland
}

\begin{abstract}
Calculations of the spontaneous polarization $\left(P_{\mathrm{s}}\right)$, dielectric susceptibility $(\chi)$ and pyroelectric coefficient $(\Pi)$ of the ferroelectric films have been performed in the thermodynamic phenomenological theory framework. The Euler-Lagrange equation determining polarization dependence on the film parameters and the external electric field was solved analytically under the boundary conditions with different extrapolation lengths at two surfaces, respectively. The depolarization field contribution was taken into account in the model of short-circuited mono-domain ferroelectric film, treated as perfect insulator. The detailed analysis of the aforementioned quantities, space distribution and their average values in two cases with and without depolarization field was carried out. It was shown that the depolarization field shifts critical temperature to smaller values and the critical thickness to bigger value in comparison to those obtained without accounting the depolarization field. Meanwhile average values of $P_{\mathrm{s}}, \chi$ and $\Pi$ dependences on the film parameters and temperature are similar to the corresponding dependences obtained without accounting the depolarization field. The depolarization field was shown to flatten $P_{\mathrm{s}}, \chi$ and $\Pi$ space distributions, which have the peculiarities otherwise (e.g. small maxima in $\chi$ and $\Pi$ coordinate profiles near the film surfaces). It was shown that depolarization field influence in short-circuited film could be neglected when the film thickness or the extrapolation lengths in the boundary conditions are larger than the correlation length value.
\end{abstract}

Keywords: depolarization field, ferroelectric film, «size-driven» phase transition

Paper received 14.03.02; revised manuscript received 30.05.02; accepted for publication 25.06.02.

\section{Introduction}

Depolarization field plays an important role in physics of ferroelectrics because it tends to destroy spontaneous electric polarization that is known to be an order parameter of these systems. The domain structure formation and the presence of free carriers are the main internal factors that tend to decrease this field influence. External condition of short-circuited ferroelectrics results in the complete compensation of the depolarization field for the bulk material. But it is not the case for the thin ferroelectric film [1] because of its inhomogeneous polarization related to the contribution of surface effects. Moreover, there is an essential difference between the domain structure of the bulk material and that of the film (see e.g. [2], [3]), namely, the thinner the film the better conditions of mono-domain state appearance. On the other hand, mono-domain ferroelectric films self-polarized in the direction normal to the surface can be obtained under special technological conditions [4]. Taking into consideration that in many cases the conductivity of ferroelectrics is small enough (see e.g. [5]) it is reasonable to consider the model of the depolarization field influence, which is proposed in [1]: mono-domain ferroelectric film under short-circuit conditions, ferroelectric treated as perfect insulator. The consideration in [1] was carried out in the phenomenological Ginzburg - Landau theory approach with polarization values on both film surfaces supposed to be the same, although in general case they have to be different. Another disadvantage of this work is related to the fact that only the ferroelectric phase polarization in the thick film (where the average polarization is equal to the bulk material value) is studied.

In our work, we performed the calculation in the same model but without the aforementioned limitations. Keeping in mind that the most part of the works published in the previous years have been performed without taking into consideration the depolarization field influence (see e.g. [6] 
- [8]) here we represent the detailed comparison of the ferroelectric film $P_{\mathrm{s}}, \chi$ and $\Pi$ space distributions and the average values obtained within the framework of models with and without taking into consideration depolarization field. This made it possible to clear up the cases and conditions at which the depolarization field can be neglected qualitatively or even quantitatively.

\section{Basic equations}

Let us consider thin ferroelectric film polarized along $\mathrm{z}$ axis (i.e. $P_{\mathrm{z}} \neq 0, P_{\mathrm{x}}=P_{\mathrm{y}}=0$ ), which is perpendicular to the film surface. This type of polarization can appear as a result of self-polarization of a film grown under special technological condition without application of an external electric field [4].

An equilibrium value of polarization can be obtained in the framework of the phenomenological thermodynamic theory from the minimum of the functional for free energy [9]. In the considered case of polarization perpendicular to the surface of the film, it is necessary to take into account depolarization field, which is proportional to the value of polarization and has the opposite direction, so it destroys $P_{\mathrm{s}}$. In accepted model ferroelectrics is regarded as a perfect insulator under short-circuit conditions. Taking into account the symmetry of considered structure (i.e. the film polarization depends only on the coordinate z) one can write the free energy density functional as follows:

$F=\frac{1}{1} \int_{0}^{1}\left[\frac{\alpha}{2} P_{z}^{2}(z)+\frac{\beta}{4} P_{z}^{4}(z)+\frac{\gamma}{2}\left(\frac{d P_{z}(z)}{d z}\right)^{2}-E_{z} P_{z}(z)\right] d z+$

$+\frac{\gamma}{21}\left(\frac{P_{z}^{2}(0)}{\delta_{1}}+\frac{P_{z}^{2}(1)}{\delta_{2}}\right)+2 \pi\left(\frac{1}{1} \int_{0}^{1} P_{z}^{2}(z) d z-\left(\frac{1}{1} \int_{0}^{1} P_{z}(z) d z\right)^{2}\right)$

Here, $l$ is thickness of the film, the coefficient a depends on temperature T as $\alpha=\alpha_{0}\left(T-T_{\mathrm{c}}\right), T_{\mathrm{c}}$ is the temperature of the transition from paraelectric to ferroelectric phase in bulk ferroelectrics, $E_{\mathrm{z}}$ is the external electric field, $\delta_{1,2}$ are extrapolation lengths. The last two and previous two terms represent depolarization field energy and surface one, respectively. In what follows, we will consider the case of positive extrapolation lengths, when polarization on the film surface is smaller than in the film centre. Only in this case surface effects can lead to the size-driven phase transition [7].

The minimization of the functional (1) results in the following equation determining the space distribution of polarization over the film thickness:

$$
\alpha P_{z}+\beta P_{z}^{3}-\gamma \frac{d^{2} P_{z}}{d z^{2}}=E_{z}-4 \pi P_{z}+4 \pi \frac{1}{1} \int_{0}^{1} P_{z} d z
$$

with boundary conditions on film surfaces:

$$
\left(\delta_{1} \frac{d P_{z}}{d z}-P_{z}\right)_{z=0}=0, \quad\left(\delta_{2} \frac{d P_{z}}{d z}+P_{z}\right)_{z=1}=0
$$

The last two terms in Eq. (2a) represent the depolarization field:

$$
E_{z}^{d}=-4 \pi\left(P_{z}-\frac{1}{1} \int_{0}^{1} P_{z} d z\right)
$$

Here, the first term is depolarization field for the freestanding film, and the second one is the compensating field of free charges on the short-circuiting electrodes. It is evident that depolarization field (3) equals to zero, when the space distribution of polarization is homogeneous.

\section{Paraelectric phase}

The equation ( $2 a$ ) for the space distribution of polarization is a non-linear inhomogeneous integral differential equation, and it is cumbersome to solve it analytically. But in paraelectric phase, where $P_{\mathrm{s}}$ equals to zero and polarization is proportional to external field, the nonlinear term in Eq. (2a) can be negligibly small.

Therefore, the solution of this linear equation can be expressed in terms of elementary functions, so subject to boundary conditions ( $2 \mathrm{~b}$ ) the space distribution of polarization in this case has the following form:

$$
\begin{gathered}
P^{P E}(\xi)=\frac{1-\varphi(\xi)}{4 \pi(\Phi-f)} E \\
\varphi(\xi)=\frac{\left(1+d_{2}-\left(1-d_{1}\right) \exp (-h)\right) \exp (-\xi)+\left(1+d_{1}-\left(1-d_{2}\right) \exp (-h)\right) \exp (\xi-h)}{\left(1+d_{1}\right)\left(1+d_{2}\right)-\left(1-d_{1}\right)\left(1-d_{2}\right) \exp (-2 h)} \\
\Phi=\langle\varphi\rangle=\frac{1-\exp (-h)}{h} \frac{2+d_{1}+d_{2}-\left(2-d_{1}-d_{2}\right) \exp (-h)}{\left(1+d_{1}\right)\left(1+d_{2}\right)-\left(1-d_{1}\right)\left(1-d_{2}\right) \exp (-2 h)}
\end{gathered}
$$


Here all indexes "z" is omitted, angle bracket means zcoordinate averaging, and the following designations are introduced:

$$
\begin{gathered}
\xi=\sqrt{1-f} \frac{z}{1_{d}} f=\frac{-\alpha}{4 \pi} 1_{d}^{2}=\frac{\gamma}{4 \pi} \\
d_{i}=\sqrt{1-f} \frac{\delta_{i}}{1_{d}} h=\sqrt{1-f} \frac{1}{1_{d}}
\end{gathered}
$$

The average polarization of the film can be easily derived from(4):

$$
\left\langle P^{P E}\right\rangle=\frac{1-\Phi}{4 \pi(-f+\Phi)} E
$$

Note that in the case $d_{1}=d_{2}$ the space distribution of polarization in paraelectric phase (4) and its average value (6) coincides with those obtained earlier [1].

The derivative $\chi=\left.(\mathrm{d} P / \mathrm{d} E)\right|_{E=0}$ represents linear dielectric susceptibility of the film. The analysis of the expression (6) has shown that value of $\chi$ is positive when either $f<0$ (i.e. $\alpha>0, T>T_{\mathrm{c}}-$ paraelectric phase of bulk ferroelectrics) or $f>0$ and $\Phi>f\left(T<T_{\mathrm{c}}\right.$ - ferroelectric phase of bulk ferroelectrics, but paraelectric phase of ferroelectric film). When equality $\Phi=f$ is valid, the value of $\chi$ becomes infinitely large, so the considered system undergoes a phase transition.

Because of $f$ and $\Phi$ values dependence on thickness and temperature, equation $\Phi=f$ determines critical temperature $T_{\mathrm{cl}}$ at fixed thickness or critical thickness $l_{\mathrm{c}}$ at fixed temperature.

The parameter $f$ value can be evaluated as $1 / \varepsilon$, where $\varepsilon$ is the dielectric permittivity of bulk ferroelectrics in paraelectric phase. Therefore, it is much smaller than unity, so the equation determining the phase transition point can be rewritten as follows:

$$
h=\frac{U_{1}+U_{2}}{f}, \quad f<<1, \quad U_{i}=\frac{1}{1+d_{i}}
$$

From this equation, it is easy to obtain expressions for critical thickness

$$
\begin{aligned}
& l_{c}=\frac{U_{1}+U_{2}}{f \sqrt{1-f}} l_{d}= \\
& =\frac{4 \pi}{-\alpha} \sqrt{\frac{\gamma}{4 \pi+\alpha}}\left(\left(\delta_{1} \sqrt{\frac{4 \pi+\alpha}{\gamma}}+1\right)^{-1}+\left(\delta_{2} \sqrt{\frac{4 \pi+\alpha}{\gamma}}+1\right)^{-1}\right)
\end{aligned}
$$

and for critical temperature owing to condition $f<<1$ :

$$
T_{c l}=T_{c}-\frac{\gamma}{\alpha_{0} l}\left(\left(\delta_{1}+\sqrt{\frac{\gamma}{4 \pi}}\right)^{-1}+\left(\delta_{2}+\sqrt{\frac{\gamma}{4 \pi}}\right)^{-1}\right)
$$

or in more visual form:

$$
\begin{aligned}
& T_{c \mathrm{l}}=T_{c}\left(1-\frac{1_{0}^{2}(0)}{1}\left(\frac{1}{\delta_{1}+l_{d}}+\frac{1}{\delta_{2}+l_{d}}\right),\right. \\
& l_{0}(0)=\sqrt{\frac{\gamma}{\alpha_{0} T_{c}}} .
\end{aligned}
$$

Here, $l_{0}(0)$ is the correlation length at zero temperature. As it follows from Eq. (9b), if the thickness value is less than $l_{\mathrm{c}}(0)=l_{0}(0)^{2} /\left(\left(\delta_{1}+l_{\mathrm{d}}\right)^{-1}+\left(\delta_{2}+l_{\mathrm{d}}\right)^{-1}\right)$, then critical temperature becomes negative, i.e. phase transition vanishes.

Therefore, ferroelectric phase transition in a film can be achieved by changing the film thickness at some fixed temperature or by changing the temperature of the film with fixed thickness. As a matter of fact, the curve described by equations $(9 \mathrm{a}, \mathrm{b})$ determine phase boundaries between paraelectric and ferroelectric phases, i.e. the phase diagram in coordinates temperature - thickness of the film, which is depicted in Fig. 1 by solid curves for the different values of the extrapolation lengths, critical thickness value $l_{\mathrm{c}}(0)$ being the cross-point of the curves with abscissa axis. Namely, at $l<l_{c}(0)$ paraelectric phase (polarization $P_{s}=0$ ) exists in all temperature region, while at $l>l_{\mathrm{c}}(0)$ it can be both ferroelectric phase $\left(P_{\mathrm{s}} \neq 0\right)$ at $T<T_{\mathrm{cl}}$ and paraelectric phase at $T>T_{\mathrm{cl}}$.

It is seen from Fig. 1 that depolarization field decreases critical temperature and increases critical thickness, these effects being stronger under decreasing the extrapolation

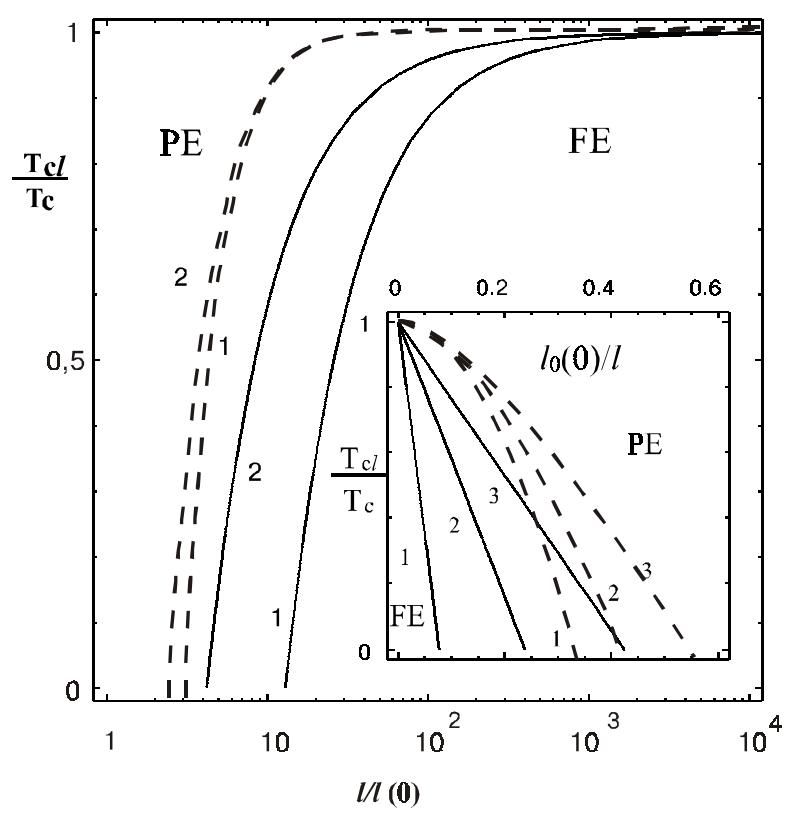

Fig. 1. The dependence of the critical temperature $T_{\mathrm{cl}}$ on the film thickness in the logarithmic scale for $f(0)=\alpha_{0} T_{\mathrm{c}} / 4 \pi=0.01$ and for different extrapolation length values: $\left(\delta_{1} / l_{\mathrm{d}}, \delta_{2} / l_{\mathrm{d}}\right)=(0.5,0.6)$, $(5,3),(10,6)$ for lines 1,2 and 3 , respectively, with (solid lines) and without (dashed lines) the depolarization field contribution. The same dependences are depicted on the inset for the inverse film thickness. 
M.D. Glinchuk et al.: The influence of depolarization field...

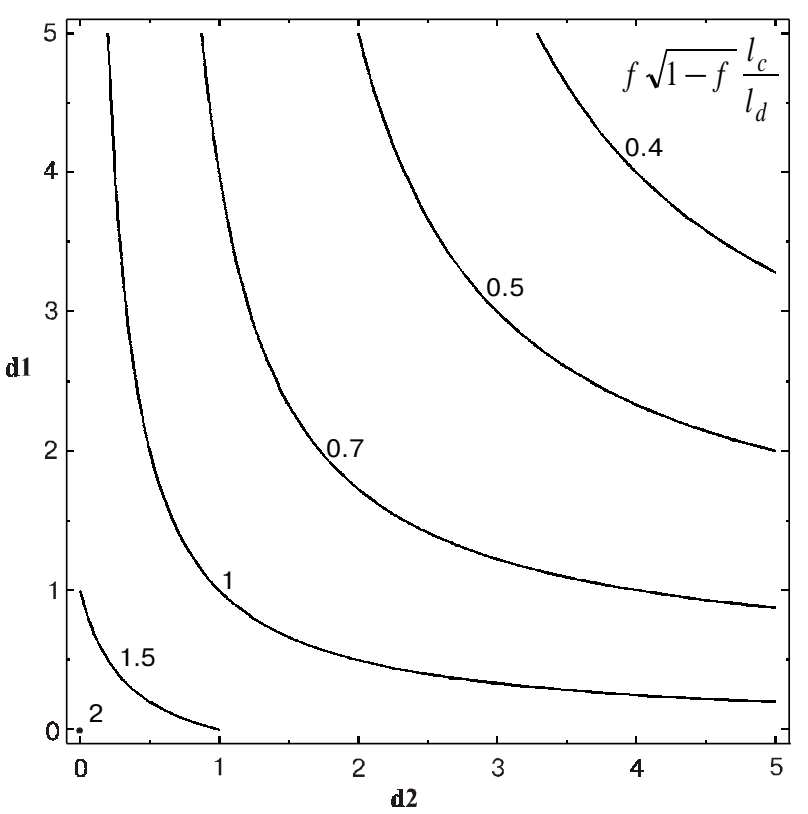

Fig. 2. The dimensionless critical thickness $f \sqrt{1-f} l_{c} / l_{d}$ given by Figs near curves dependence on the dimensionless extrapolation lengths $d_{\mathrm{i}}$.

lengths values (compare solid and dashed curves in Fig.1). More detailed critical thickness value dependence on the extrapolation lengths values is depicted in Fig. 2. One can see that the greater $d_{1}$ and $d_{2}$ values the smaller $l_{\mathrm{c}}$ value. The latter can be related to flattening the polarization space distribution with increasing the values of extrapolation lengths that results in the critical thickness decrease and increase of critical temperature (compare curves 1, 2 and 3 in Fig.1).

When inequalities $\Phi<f$ and $f>0$ are valid, polarization determined by equation (4) becomes negative, so nonlinearity in equation ( $2 a$ ) cannot be neglected.

\section{Ferroelectric phase}

\subsection{Free energy}

In ferroelectric phase, i.e. in the region $l>l_{\mathrm{c}}(0)$ and $T<T_{c l}$ non-linearity needs to be taken in consideration. The simplest way to take it into account is the direct variational method. We choose the solution (4) as a trial function and an amplitude factor will be treated as a variational parameter. It should be noted, in ferroelectric phase (i.e. under condition $\left.h \geq\left(U_{1}+U_{2}\right) / f\right)$ and in the case of small $f$ values one can suggest $h>>1$, so the expressions for the function $\varphi(\xi)$ can be essentially simplified. Therefore, we will look for the polarization space distribution in ferroelectric phase in the following form:
$P^{\mathrm{FE}}(\xi)=P(1-\alpha(\xi))$

$\varphi(\xi)=\left\{\begin{array}{cc}U_{1} \exp (-\xi), & 0 \leq \xi<<h / 2 ; \\ U_{2} \exp (\xi-h), & 0 \leq h-\xi<<h / 2 ;\end{array}\right.$

Where $P$ is the variational parameter that represents the amplitude of the polarization space distribution.

After integration of the free energy density (1) with trial function (10), one can easily obtain free energy density as follows:

$$
F=\alpha\left(1-\frac{A_{1}}{f}\right)\left(1-A_{1}\right) \frac{P^{2}}{2}+\beta(1-B) \frac{P^{4}}{4}-E P\left(1-A_{1}\right)
$$

Keeping in mind that the polarization average value is $\langle P\rangle=P\left(1-A_{1}\right)$, one can rewrite Eq.(11a) in the form:

$$
F=\alpha \frac{\left(1-A_{1} / f\right)}{1-A_{1}} \frac{\langle P\rangle^{2}}{2}+\frac{\beta(1-B)}{\left(1-A_{1}\right)^{4}} \frac{\langle P\rangle^{4}}{4}-E\langle P\rangle
$$

Here the following designation are used

$A_{1}=\frac{U_{1}+U_{2}}{h}, \quad B=\frac{G\left(U_{1}\right)+G\left(U_{2}\right)}{12 h}$,

$G(U)=48 U-36 U^{2}+16 U^{3}-3 U^{4}$

Coefficients in the free energy written as power series over the polarization amplitude $\mathrm{P}$ (11a) or the polarization average value $\langle\mathrm{P}\rangle$ (11b) depend not only on the temperature but on the film thickness and extrapolation lengths, too. We found that the dependence of the coefficient of polarization to the fourth power on the film thickness and temperature is weak, while the coefficient of the second power polarization tends to zero in the size-driven phase transition point, i.e. when reaching the critical temperature or thickness.

Note that for bulk ferroelectrics, i.e. in the case the film thickness tending to infinity, it is seen from Eq.(12) that quantities $A_{1}$ and $B$ tend to zero, and hence the free energy densities both (11a) and (11b) tend to well known expression for free energy $F=\alpha P^{2} / 2+\beta P^{4} / 4-E P$. It is evident that the difference between this expression and Eq.(11b) can be reduced to the renormalization of coefficients $\alpha$ and $\beta$, but free energy (11) consists of terms, which are determined by the correlation energy, surface energy and depolarization field energy. All these quantities are contained in the free energy density (1) of the considered system and mainly reduced to the first term in the free energy (11). Therefore, the free energy functional (1) is reduced to the conventional free energy form (11) allowing for Eq. (10) determining the polarization space distribution in the ferroelectric film.

It should be noted that Eq. ( $2 a$ ) could be linearized and easily solved analytically in two important cases: in the vicinity of the phase transition, where $P_{\mathrm{s}}$ tends to zero and can be considered as the small parameter, and 
M.D. Glinchuk et al.: The influence of depolarization field...

for sufficiently thick films, when the polarization space distribution is almost uniform. The solutions obtained by these methods coincide with the solution obtained by the variational method in the above-mentioned limits.

\subsection{Spontaneous polarization}

The equation determining the relation between the maximal value of the polarization and external field, temperature, film thickness and other parameters of the considered system can be easily obtained by minimization of Eq. (11a) on parameter $P$. Despite the analytical solution of this equation exists for arbitrary external field value, it is rather cumbersome and difficult to analyze it.

Hereinafter we consider in details two physically meaningful cases: zero external field and small external field value.

In the first case one can find following expression for the space distribution of spontaneous polarization $P_{\mathrm{s}}=P(E=0)$ and its average value:

$$
\begin{aligned}
& P_{\mathrm{s}}(\xi)=\mathrm{P}_{s 0} \sqrt{\frac{1-A_{1}}{1-B}\left(1-\frac{A_{1}}{f}\right)}(1-\varphi(\xi)) \\
& \left\langle P_{\mathrm{s}}\right\rangle=P_{\mathrm{s} 0} \sqrt{\frac{\left(1-A_{1}\right) 3}{1-B}\left(1-\frac{A_{1}}{f}\right)}
\end{aligned}
$$

Where $P_{\mathrm{s} 0}=\sqrt{-\frac{\alpha}{\beta}}$ is the homogeneous spontaneous po-

larization of bulk ferroelectrics at $T \leq T_{\mathrm{c}}$. As it follows from these expression, $P_{\mathrm{s}}$ tends to zero when it reaches the phase transition point and exists only under condition $f \geq A_{1}=\left(U_{1}+U_{2}\right) / h$, as it should be suggested. The expressions (13a) with respect to Eqs. (5), (7), (12) determine $P_{\mathrm{s}}$ space distribution and average value dependence on the film thickness and temperature.

Average value of $P_{\mathrm{s}}$ can be simplified in the vicinity of ferroelectric phase transition and for the films with large thickness. In these regions $P_{\mathrm{s}}$ dependence on the film thickness can be written in the simple form:

$$
\frac{\left\langle P_{s}\right\rangle}{P_{s_{0}}} \sim \sqrt{1-\frac{l_{c}}{l}}, \quad l-l_{c}<<l_{c} ; \quad \frac{\left\langle P_{s}\right\rangle}{P_{s_{0}}}-1 \sim \frac{l_{c}}{2 l}, \quad l>>l_{c} ;
$$

Here proportionality coefficients are omitted because of their cumbersome form and weak dependence on the temperature and extrapolation lengths. Notice, that their value are close to unity. For the arbitrary film thickness value, the polarization has to be calculated using Eqs (13a).

The space distribution of $P_{\mathrm{s}}$ (the scale $\mathrm{z} / l_{0}$, here $l_{0}=\sqrt{-\frac{\gamma}{\alpha}}$ is the correlation length at $T \leq T_{\mathrm{c}}$ ) and its av-

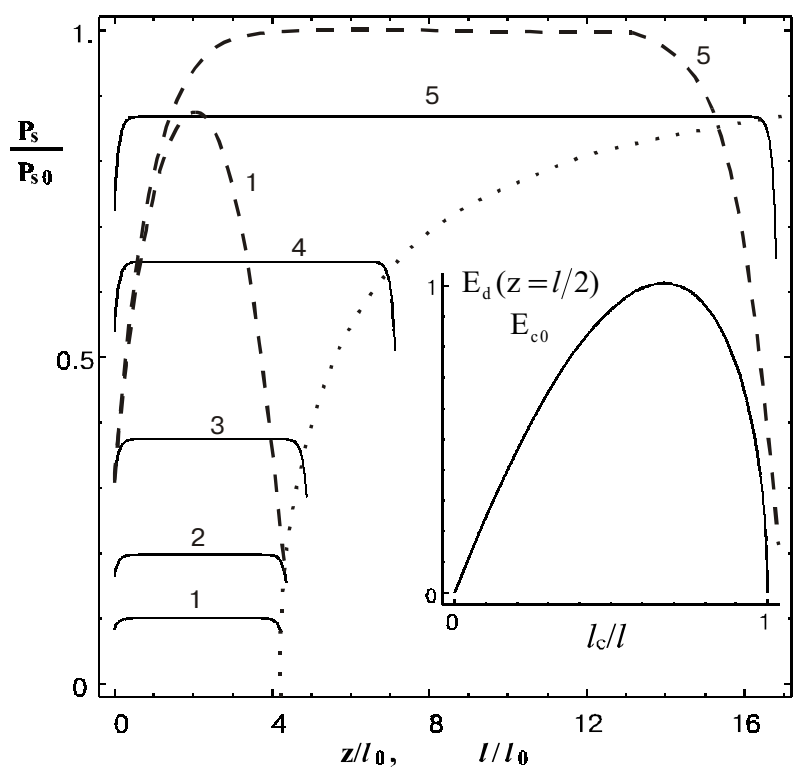

Fig. 3. The space distribution of $P_{\mathrm{s}}$ over $\mathrm{z}$ coordinate for the following values of parameters: $f=0.01, \delta_{1} / l_{\mathrm{d}}=5, \delta_{2} / l_{\mathrm{d}}=3$, and for the different values of the film thickness $l / l_{\mathrm{c}}=1.01,1.04,1.16,1.7$, 4 (solid curves $1-5$ respectively). Dashed curves 1 and 5 represent the space distribution of $P_{\mathrm{s}}$ calculated without taking the depolarization field into account. The average value of $P_{\mathrm{s}}$ over the film thickness $l$ is represented by dotted curve. The depolarization field in the center of the film as a function of the inverse film thickness is depicted on the inset for parameter values given above.

erage value dependence on the film thickness (the scale $l /$ $l_{0}$ ) is depicted in Fig. 3 by solid curves and dotted curve, respectively, for the different values of parameters. It can be easily seen that the polarization space distribution remains smooth in the most part of the film even for the thin film with thickness in the vicinity of critical value $l_{\mathrm{c}}$ (see solid curves 1 and 2), where $P_{\mathrm{s}}$ tends to zero. This phenomenon can be explained by the influence of the depolarization field that tends to flatten the polarization space distribution (compare the solid and dashed lines).

One can see also that depolarization field influence depends on the film thickness: the thinner the film the larger the depolarization field value. To illustrate this phenomenon, in the inset to Fig. 3 we represented the dependence of the ratio of the depolarization field in the film center to the coercive electric field $E_{\mathrm{c} 0}$ for the bulk ferroelectrics $E_{c 0}=\frac{-8 \pi f}{(3 \sqrt{3}) P_{s 0}}$ on the film thickness. It is seen that depolarization field increases when the film thickness decreases up to a maximum value, that is approximately equal to the coercive electric field, and then abruptly decreases to zero in phase transition point.

The space distribution of the depolarization field (solid curves) and $P_{\mathrm{s}}$ (dashed curves) in the vicinity of film surface is represented in Fig. 4. As it is shown in this picture, these quantities deviate from the homogeneous 


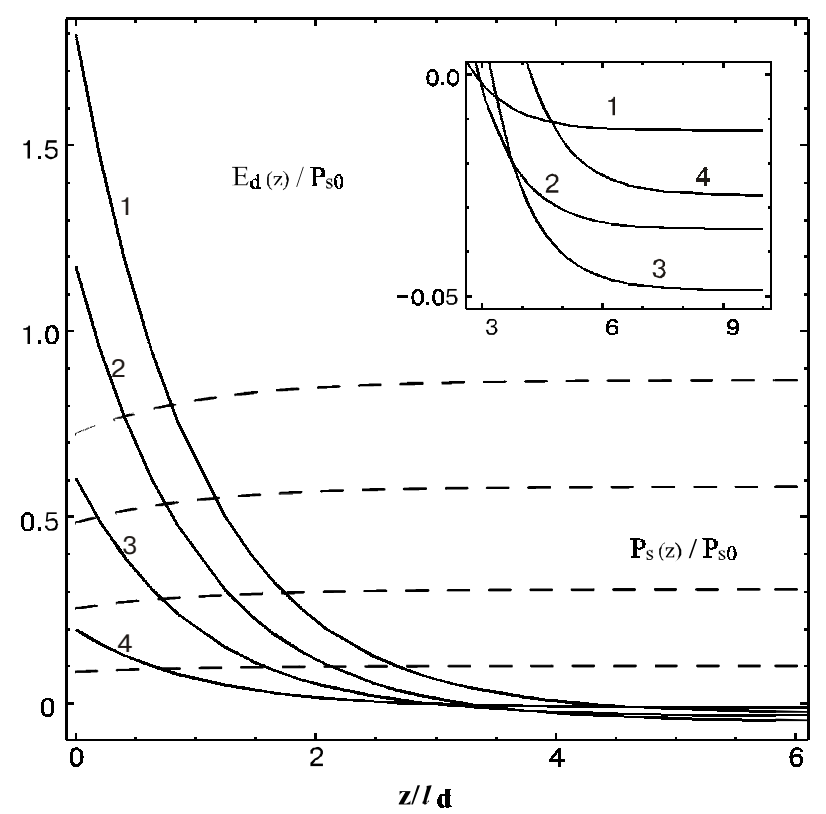

Fig. 4. The space distribution of the depolarization field (solid curves) and $P_{\mathrm{S}}$ (dashed curves) in the vicinity of film surface for the following values of parameters: $f=0.01, \delta_{1} / l_{\mathrm{d}}=5, \delta_{2} / l_{\mathrm{d}}=3$, and for different values of the film thicknesses $l / l_{\mathrm{c}}=1.01,1.1,1.5,4$ (curves 1 to 4 , respectively). The region of the plot where the depolarization field is negative is depicted on the inset.

space distribution within the range of $l_{\mathrm{d}}$ order related to the strength of correlation energy in the free energy density (1). It is also seen that in this region depolarization field has the same direction as polarization, but with increasing the distance from the surface depolarization field becomes negative (see inset to Fig.4) This behavior explains the flattening and decreasing of the polarization caused by depolarization field (compare the solid and dashed curves in Fig. 3).

The dependence of $P_{\mathrm{s}}$ average value on temperature is shown in Fig. 5. One can see that this dependence general form undergoes minor changes in comparison with that of bulk material polarization, but only the critical temperature value is shifted with changing the film thickness.

\subsection{Dielectric susceptibility and pyroelectric coefficient}

In the case of small external electric field one can obtain linear dielectric susceptibility $\chi=\left.(\mathrm{d} P / \mathrm{d} E)\right|_{E=0}$ of the film in ferroelectric phase from the free energy density (11):

$\chi^{\mathrm{FE}}(\xi)=\chi_{0} \frac{f(1-\varphi(\xi))}{f-A_{1}},\left\langle\chi^{\mathrm{FE}}\right\rangle=\chi_{0} \frac{f\left(1-A_{1}\right)}{f-A_{1}}$

Here, $\chi_{0}=1 /(-2 \alpha)$ is dielectric susceptibility of bulk ferroelectrics at $T \leq T_{\mathrm{c}}$. The value of $\chi(14)$ becomes infinitely large in the phase transition point. The analysis of

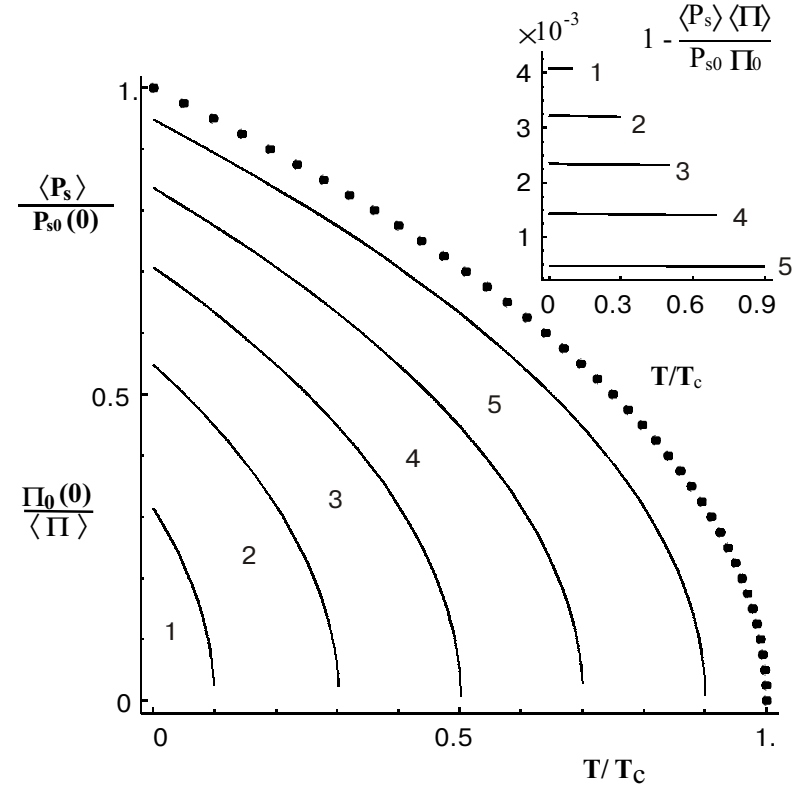

Fig. 5. The dependences of $P_{\mathrm{s}}$ average value and inverse average value of $\mathrm{P}$ on temperature for the following values of parameters: $f(0)=\alpha_{0} T_{\mathrm{c}} / 4 \pi=0.01, \delta_{1} / l_{\mathrm{d}}=5, \delta_{2} / l_{\mathrm{d}}=3$, and for the different values of the film thickness $l / l_{\mathrm{c}}(0)=1.11,1.43,2,3.33,10$ (curves 1 to 5 , respectively). The temperature dependence of the bulk ferroelectrics spontaneous polarization $P_{\mathrm{s} 0}=P_{\mathrm{s} 0}(0) \sqrt{1-T / T_{c}}$ is depicted by circles. The relative difference between average value $P_{\mathrm{s}}$ and inverse average value of $P$ is depicted in the inset.

the expressions (6) and (14) have shown that on the equal distances from the critical value (not far from it) average dielectric susceptibility in paraelectric phase is two times larger than average $\chi$ in ferroelectric phase like that for bulk ferroelectrics

Average value of $\chi$ can be simplified in the vicinity of ferroelectric phase transition and for the films with large thickness. In these regions $\chi$ dependence on the film thickness can be written in the simple form:

$\frac{\left\langle\chi^{F E}\right\rangle}{\chi_{0}}=\frac{1-f}{1-l_{c} / l}, l-l_{c}<<l_{c} ;$

$\frac{\left\langle\chi^{F E}\right\rangle}{\chi_{0}}=1+(1-f) \frac{l_{c}}{l}, l>>l_{c}$;

For the arbitrary film thickness value $\chi$ has to be calculated on the base of Eqs (14a).

The space distribution of $\chi$ in ferroelectric phase over $z$ coordinate (the scale $z / l_{0}$ ) and its average value dependence on the film thickness (the scale $l / l_{0}$ ) is depicted in Fig. 6 by solid curves and dotted curve, respectively. Comparison of dashed and solid curves shows that the depolarization field presence increases $\chi$ value for the thin films in the vicinity of the critical thickness value 
M.D. Glinchuk et al.: The influence of depolarization field...

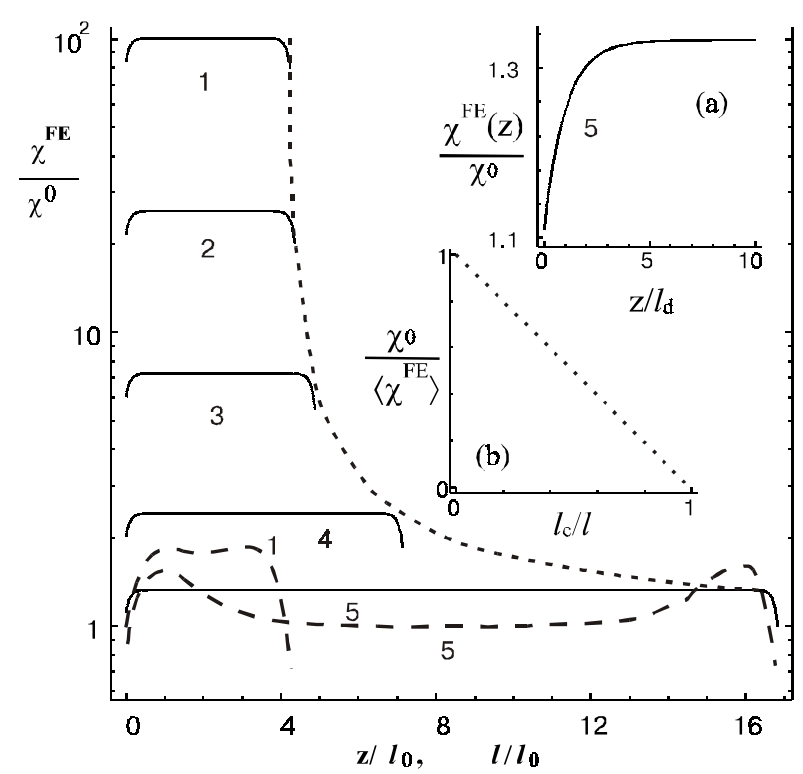

Fig. 6. The space distribution of $\chi$ in ferroelectric phase over $z$ coordinate for the following values of parameters: $f=0.01, \delta_{1}$ I $l_{\mathrm{d}}=5, \delta_{2} / l_{\mathrm{d}}=3$, and for the different values of the film thickness $/ /$ $l_{\mathrm{c}}=1.01,1.04,1.16,1.7,4$ (solid curves 1 to 5 , respectively). Dashed curves 1 and 5 represent $\mathrm{c}$ space distribution calculated without taking the depolarization field into account. The average value of $\chi$ over the film thickness $l$ is represented by the dotted curve. The space distribution of $\chi$ in the vicinity of film surface is depicted in the inset (a) for the film thickness value, given above for curve 5 . The inset (b) represents the dependence of the inverse average $\chi$ on the inverse thickness of the film.

(see curves 1). But for the films with thickness several times larger, $\chi$ space distribution obtained without taking into account the depolarization field has the maxima near the surfaces (see dashed curve 5) contrary to $\chi$ space distribution obtained with taking into account the depolarization field (see solid curve 5) that remains flat. In these regions, the depolarization field decreases $\chi$ value.

It seems to us that experimental observation of $\chi$ maxima in the vicinity of film surfaces could be considered as manifestation of the depolarization field complete compensation. On the other hand the flatness of $\chi$ space distribution (like solid curves in Fig. 6) gives evidence of depolarization field presence. More detailed form of $\chi$ space distribution near the film surface for this case is shown in the inset (a) to Fig. 6. The dotted line in

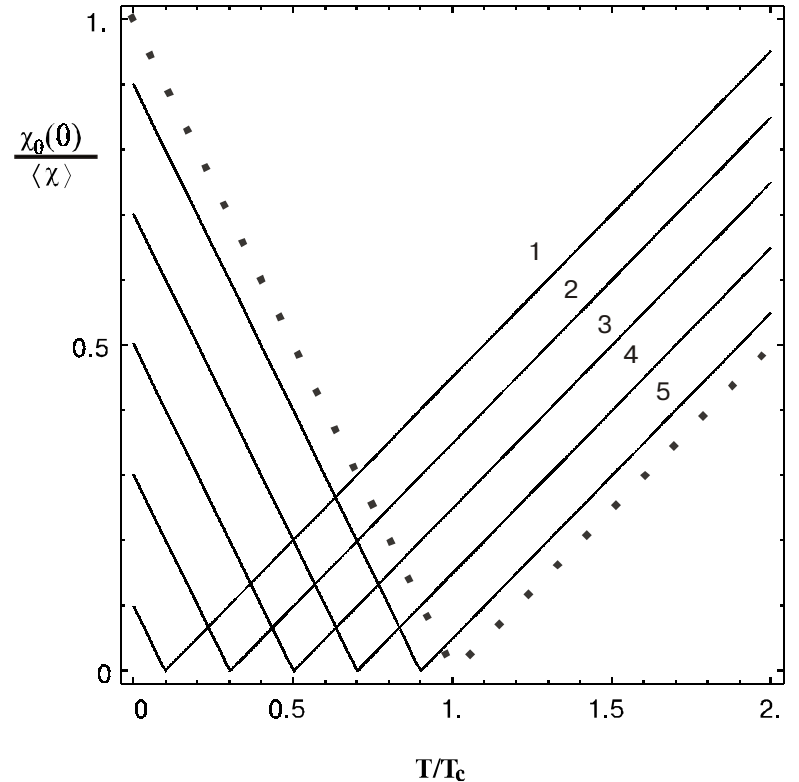

Fig. 7. The dependence of the inverse average value of $\chi$ on temperature for the following values of parameters: $f(0)=\alpha_{0} T_{\mathrm{c}}$ l $4 \pi=0.01, \delta_{1} / l_{\mathrm{d}}=5, \delta_{2} / l_{\mathrm{d}}=3$, and for the different values of the film thickness $l / l_{\mathrm{c}}(0)=1.11,1.43,2,3.33,10$ (curves 1 to 5 , respectively). Squares depict the temperature dependence of the bulk ferroelectrics inverse dielectric susceptibility $\mathrm{c}_{0}(\mathrm{~T})$.

the inset (b) reflects the linear dependence of the inverse average $\chi$ on the inverse film thickness value in all the region $l \geq l_{\mathrm{c}}$ and not just in the regions $l>>l_{\mathrm{c}}$ and $l \approx l_{\mathrm{c}}$ as it is shown in Eqs (14b).

The dependence of the inverse average value of $\chi$ on temperature is represented in Fig. 7. One can see that temperature dependence of the film average $\chi$ resemble that of the bulk materials with the critical temperature $\mathrm{T}_{\mathrm{cl}}$ value being dependent on the film thickness and presence or absence of depolarization field as it is shown in Fig.1.

Pyroelectric coefficient $\Pi=\mathrm{d} P_{\mathrm{s}} / \mathrm{d} T$ can be obtained by direct differentiation of the corresponding expression for polarization on temperature. In addition it is necessary to take into account the dependence of the parameter $f$ on temperature (see definitions (5)) and the dependence of other parameters on $f$ (see equations (7), (10) and (12)).

One can obtain from Eq. (13) the following expression, determining the space distribution of $\Pi$ :

$$
\begin{gathered}
\frac{\Pi(\xi)}{\Pi_{0}}=\left(1+\frac{2 A_{1}\left(A_{1}-1\right)}{1-f}+\frac{A_{2}\left(1+f-2 A_{1}\right)}{2(1-f)}+\frac{C\left(1-A_{1}\right)\left(f-A_{1}\right)}{2(1-f)(1-B)}\right) \frac{1-\varphi(\xi)}{\sqrt{\left(1-A_{1}\right)\left(1-A_{1} / f\right)(1-B)}}- \\
-\frac{f}{1-f} \sqrt{\frac{1-A_{1}}{1-B}\left(1-\frac{A_{1}}{f}\right) \varphi_{d}(\xi)}
\end{gathered}
$$


M.D. Glinchuk et al.: The influence of depolarization field...

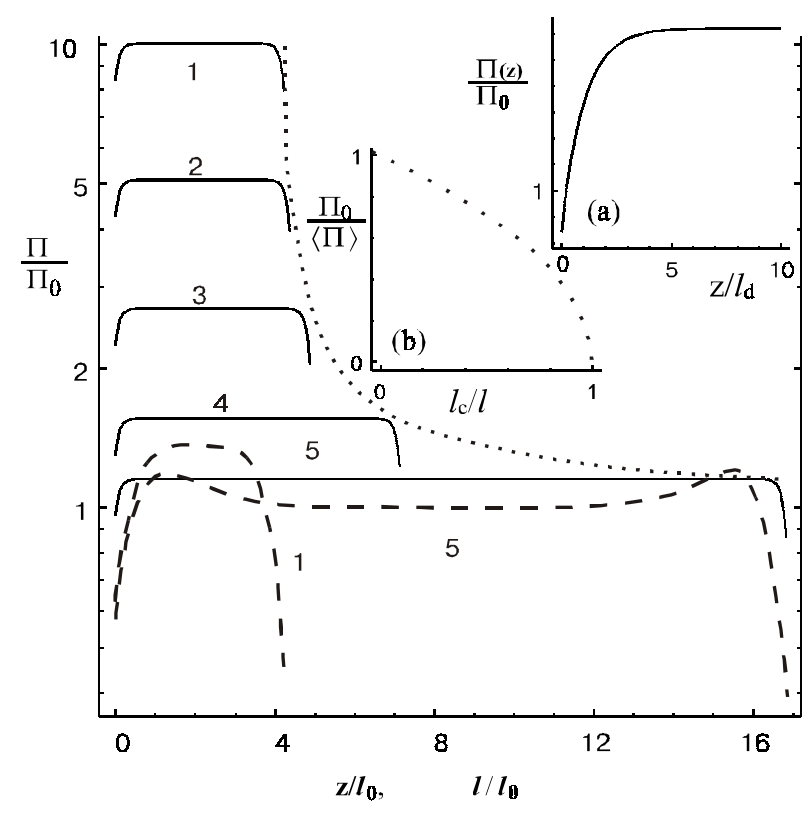

Fig. 8. The space distribution of $P$ over $z$ coordinate for the following values of parameters: $f=0.01, \delta_{1} / l_{\mathrm{d}}=5, \delta_{2} / l_{\mathrm{d}}=3$, and for the different values of the film thickness $l / l_{\mathrm{c}}=1.01,1.04,1.16,1.7$, 4 (solid curves 1 to 5 , respectively). Dashed curves 1 and 5 represent $\Pi$ space distribution calculated without taking the depolarization field into account. The average value of $\Pi$ over the film thickness $l$ is represented by the dotted curve. The space distribution of $\Pi$ in the vicinity of film surface is depicted in the inset (a) for the film thickness value given above for the curve 4 . The inset (b) represents the dependence of the inverse average $\mathrm{P}$ on the inverse film thickness.

Here, $\Pi_{0}=\mathrm{d} P_{\mathrm{s} 0} / \mathrm{d} T$ is the pyroelectric coefficient of bulk ferroelectrics below critical temperature $T<T_{\mathrm{c}}$, the following designation are used

$A_{2}=\frac{U_{1}^{2}+U_{2}^{2}}{h}, \quad C=\frac{V\left(U_{1}\right)+V\left(U_{2}\right)}{12 h}$,

$V(U)=96 U-156 U^{2}+136 U^{3}-63 U^{4}+12 U^{5}$

and the following function is introduced:

$\varphi_{d}(\xi)=\left\{\begin{array}{cc}U_{1}\left(1-U_{1}+\xi\right) \exp (\xi), & 0 \leq \xi<<h / 2 ; \\ \left.U_{2}\left(1-U_{2}+h-\xi\right) \exp \xi-r\right), & 0 \leq h-\xi<<h / 2 ;\end{array}\right.$

The average value of $\Pi$ one can be easily obtain from Eq. (15a):

$$
\begin{aligned}
& \frac{\langle\Pi\rangle}{\Pi_{0}}=\sqrt{\frac{f\left(1-A_{1}\right)}{\left(f-A_{1}\right)(1-B)}} \times \\
& \times\left(1+\frac{2 A_{1}\left(2 A_{1}-1-f\right)}{1-f}+\frac{A_{2}\left(1+3 f-4 A_{1}\right)}{2(1-f)}+\frac{C\left(1-A_{1}\right)\left(f-A_{1}\right)}{2(1-f)(1-B)}\right)
\end{aligned}
$$

Average value of $\Pi$ can be simplified in the vicinity of ferroelectric phase transition and for the films with large thickness. In these regions, $\Pi$ dependence on the film thickness one can find in the form:

$$
\frac{\langle\Pi\rangle}{\Pi_{0}} \sim \sqrt{\frac{1}{1-l_{c} / l}}, \quad l-l_{c}<<l_{c} ; \quad \frac{\langle\Pi\rangle}{\Pi_{0}}-1 \sim \frac{l_{c}}{2 l}, \quad l>>l_{c} ;
$$

Here, proportionality coefficients are omitted because of their cumbersome form and weak dependence on the temperature and extrapolation lengths. Notice, that their value are close to unity.

The space distribution of $\Pi$ over $z$ coordinate and its average value dependence on the film thickness are depicted in Fig.8. The dependence of the inverse average value of $\Pi / \Pi_{0}$ on temperature practically coincides with the dependence of average value of $P_{\mathrm{s}} / P_{\mathrm{s} 0}$ (see Fig.5 and inset to it). One can see that qualitatively the behaviour of $\Pi$ space distribution and average value resembles that of $\chi$, but $\Pi$ diverges at the phase transition point with a critical index -1/2 (see Eqs (15) - (16) and inset (b) in Fig. 8), $\chi$ diverges but with a critical index -1 (see Eqs (14a), (14b) and inset (b) in Fig. 6).

Therefore, the behavior of the average quantities, including proportionality of the inverse average value of $\Pi$ to the average value of $P_{\mathrm{s}}$, is similar to that in bulk materials. This phenomenon may be the consequence of coincidence of the free energy (11b) for $\langle P\rangle$ value with that in bulk materials, including the fact that only the coefficient before $\langle P\rangle^{2}$ in Eq. (11b) essentially depends on temperature. This coefficient and the coefficient before $\langle P\rangle^{4}$ have the strong dependence on the film thickness and extrapolation lengths (see Eqs (12), (7), (5)). This lead to the dependence of average film properties on the film characteristics.

\section{Model without taking depolarization field into consideration}

In order to discuss the depolarization field influence let us consider briefly the model without accounting the depolarization field (see dashed lines in Figs 1, 3, 6 and 8). In this case the equation determining the space distribution of polarization over the film thickness has the form Eq. (2a) without the last two terms. This equation has the nontrivial solution, when the electric field equals to zero. For the case of the boundary conditions ( $2 b$ ) with positive extrapolation lengths this solution exists only under condition $\alpha<0$ (see, e.g. [10]) and represent the following space distribution of $P_{\mathrm{s}}$ :

$$
P_{s}(z)=P_{s 0} \sqrt{\frac{2 m}{1+m} \operatorname{sn}\left(\frac{z+z_{0}}{l_{0} \sqrt{1+m}} \mid m\right)}
$$


Here, $l_{0}=\sqrt{-\frac{\gamma}{\alpha}}$ is the correlation length, $\operatorname{sn}(u \mid m)$ is

the elliptic sine function [10], and constants $m$ and $z_{0}$ have to be determined from the boundary conditions (2b). Using the properties of the elliptic functions it is easy to obtain equations, which these constants satisfy:

$l=l_{0}\left(2 K(m)-F\left(\arcsin \left(f_{1}\right), m\right)-F\left(\arcsin \left(f_{2}\right), m\right)\right)$,

$z_{0}=l_{0} F\left(\arcsin \left(f_{1}\right), m\right)$;

Where $K(m)$ and $F(\varphi, m)$ are complete and incomplete elliptic integrals respectively [10], and the following designation are introduced:

$$
\begin{aligned}
& f_{i} \equiv f\left(m, \delta_{i}\right)= \\
& =\sqrt{\frac{1+m}{2 m}\left(1+\frac{1_{0}^{2}}{\delta_{i}^{2}}-\sqrt{\left(1+\frac{1_{0}^{2}}{\delta_{i}^{2}}\right)^{2}-\frac{4 m}{(1+m)^{2}}}\right)}
\end{aligned}
$$

It should be noted that nontrivial solution of equation (2a) at temperature region above critical temperature $T>T_{\mathrm{c}}$ and zero electric field $E=0$ cannot satisfy boundary conditions (2b) with positive extrapolation lengths, so only trivial solution $P_{\mathrm{s}}=0$ can.

The parameter $m$ varies from 0 to 1 in Eqs (17) - (19), otherwise $P_{\mathrm{s}}$ were a complex number. As it follows from Eq. (18a), limit $m \rightarrow 1$ corresponds to the film with thickness $l>>l_{0}$, i.e. bulk material, and under condition $m \rightarrow 0$ the value of thickness 1 tends to some critical value $l_{\mathrm{c}}$, which corresponds to zero value of $P_{\mathrm{s}}$ space distribution (17). When thickness of the film is less than critical one, $P_{\mathrm{S}}$ is absent. The critical thickness can be expressed as:

$l_{c}=l_{0}\left(\pi-\arcsin \left(\mathrm{g}_{1}\right)-\arcsin \left(\mathrm{g}_{2}\right)\right), \mathrm{g}_{\mathrm{i}}=\frac{\delta_{i}}{\sqrt{l_{0}^{2}+\delta_{1}^{2}}}$

This equation represents the critical thickness at which size-driven ferroelectric phase transition arises, i.e. ferroelectric phase disappears in the films thinner than critical thickness $1_{c}$. Therefore, phase transition from ferroelectric phase $\left(\mathrm{P}_{\mathrm{s}} \neq 0\right)$ to paraelectric phase $\left(\mathrm{P}_{\mathrm{S}}=0\right)$ can take place with decreasing the film thickness (sizedriven phase transition) at fixed temperature below the critical temperature of bulk ferroelectrics, $T<T_{\mathrm{c}}$.

On the other hand, phase transition point can be achieved at fixed thickness $l$ by changing the film temperature, since the correlation length $l_{0}=\sqrt{\gamma / \alpha_{0}\left(T_{c}-T\right)}$ and the critical thickness owing to Eq. (20) depend on temperature. The critical temperature $T_{\mathrm{cl}}$ for the film with thickness 1 can be found from the condition $l=l_{\mathrm{c}}\left(T=T_{c l}\right)$, which gives us a transcendental equation for the dependence of $T_{c l}$ value on the film parameters. This equation can be essentially simplified for two extreme cases: for the films with small-scale thick- ness, when the phase transition temperature is much smaller than this value in bulk ferroelectrics $\left(T_{\mathrm{cl}}<<T_{\mathrm{c}}\right)$, and for the films with large thickness, when the phase transition temperature is of the same order of magnitude as this value in bulk ferroelectrics $\left(\omega_{\mathrm{cl}} \approx T_{\mathrm{c}}\right)$ :

$$
\begin{aligned}
& T_{c l}=T_{c}\left(1-\left(\frac{1_{c}(0)+1_{0}(0)\left(g_{1}(0)+g_{2}(0)\right)}{1+1_{0}(0)\left(g_{1}(0)+g_{2}(0)\right)}\right)^{2}\right) \\
& l-l_{c}(0)<<l_{0}(0)=\sqrt{\frac{\gamma}{\alpha_{0} T_{c}}}
\end{aligned}
$$

$$
T_{c l}=T_{c}\left(1-\left(\frac{\pi l_{0}(0)}{l+\delta_{1}+\delta_{2}}\right)^{2}\right), \quad l>>l_{0}(0) ;
$$

Here, $l_{c}(0)$ and $l_{0}(0)$ are the critical thickness and the correlation length at zero temperature $T=0$, $g_{\mathrm{i}}(0)=\delta_{\mathrm{i}} / \sqrt{l_{0}(0)^{2}+\delta_{i}^{2}}$.

The respective differentiation of Eq. (2a) with the last two terms omitted and Eq. (2b) on temperature and external electric field gives the differential equations and the boundary conditions determining $\Pi$ and $\chi$ space distributions, respectively. The homogeneous solution of these equations, which is the particular case of the Lame equation, is a sum of Lame functions of the first and the second kind [11]. The inhomogeneous solution of these equations can be obtained by the method of variation of constants (see [12] for details).

Average values of $P_{\mathrm{s}}, \chi$ and $\Pi$ can be simplified in the vicinity of ferroelectric phase transition and for the films with large thickness. In these regions, all these quantities dependence on the film thickness one can find in the form, similar to Eqs (13b), (14b), (16b), respectively, with proportionality coefficients being order of unity and weakly dependent on the temperature and extrapolation lengths. Note that in these equations critical thickness $l_{\mathrm{c}}$ is regarded as the quantity determined by Eq. (20).

\section{Discussion}

As it follows from the above consideration the depolarization field does not change the general form of $P_{\mathrm{s}}, \chi$ and $\Pi$ average values dependence on the film thickness and temperature. The depolarization field smoothes these quantities space distributions and, in particular, destroys the maxima near the film surfaces in the space distributions of $\chi$ and $\Pi$, obtained in the case when depolarization field can be neglected.

The main effect of the depolarization field is the shift of the boundary between paraelectric and ferroelectric phases on the phase diagram temperature - film thickness to the larger thickness value as one can see from Fig.1. This shift can be especially large for the thin film 
because critical temperature $T_{\mathrm{cl}}$ one can represent as $T_{\mathrm{c}}-T_{\mathrm{cl}} \sim 1 / l$ with depolarization field contribution (see Eqs (9) and solid lines in Fig. 1) and $T_{\mathrm{c}}-T_{\mathrm{cl}} \sim 1 / l^{2}$ without it (see Eqs (21) and dashed lines in Fig. 1). Because of this fact the scattering of critical values can be expected for the films produced in the different technological conditions and when using materials with different degree of purity. These factors can influence the domain structure and conductivity of the film and, consequently, the depolarization field.

In order to elucidate the depolarization field influence, we calculated the critical thickness value with the well-known free energy parameters of $\mathrm{BaTiO}_{3}$ (see, e.g., [8] and [13]) and for temperature and extrapolation lengths equal to zero. Critical thickness reaches its maximal value for these extrapolation lengths (see Eqs (8), (20) and Fig. 2) and equals to $10 \AA$ for the model without accounting the depolarization field and increases to $130 \AA$ with accounting this field influence. Therefore, the account of depolarization field results in the increase of the critical thickness value by an order of magnitude.

It is obvious that depolarization field in sufficiently thick films under short-circuit conditions can be neglected in the most part of the film, except the vicinity of the film surfaces. Another important case, when the contribution of the depolarization field can be neglected, is related to the conditions on the film surfaces. In particular for the extrapolation length values much larger than $l_{\mathrm{d}}$ parameter value, it follows from Eq. (8) that critical value of the film thickness $l_{\mathrm{c}}$ at fixed temperature $T<T_{\mathrm{c}}$ is defined as:

$$
\frac{l_{c}}{l_{0}(T)}=\frac{1}{1-f(T)}\left(\frac{l_{0}(T)}{\delta_{1}}+\frac{l_{0}(T)}{\delta_{2}}\right), \quad \delta_{i}>l_{d} \text {. }
$$

When considering the film with definite thickness this equation determines the critical temperature $T_{\mathrm{cl}}$ dependence on the film thickness. When we neglect the depolarization field influence, the phase transition point is determined by Eq. (20). If the extrapolation lengths values much larger than correlation length $l_{0}$ one can rewrite Eq. (20) as follows:

$$
\frac{l_{c}}{l_{0}(T)}=\frac{l_{0}(T)}{\delta_{1}}+\frac{l_{0}(T)}{\delta_{2}}, \quad \delta_{i}>l_{0}(T) .
$$

Note that when temperature $T$ tends to the critical value of bulk material $T_{\mathrm{c}}$ strong inequality in Eq. (23) becomes invalid because correlation length tends to infinity in above-mentioned limit. Therefore Eq. (23) is not valid in this temperature region, too.

Keeping in mind that parameter $f$ is much smaller than unity and in general case $l_{0}(\mathrm{~T})>>l_{\mathrm{d}}$, it is seen that Eq. (22) coincides with Eq. (23) when strong inequality $\delta_{\mathrm{i}}>>l_{0}(\mathrm{~T})$ is valid and so critical parameters have to be the same in models with and without taking into account the depolarization field.
It would be extremely desirable to check the theoretical forecast by observation of the properties of the ferroelectric film prepared with special attention to the variation of the internal factors like the domain structure and the sufficient concentration of the free carriers, which can lead to the controllable decrease of the depolarization field contribution.

\section{Acknowledgements}

The authors are grateful to Prof. Michael Karkut and Prof. N.V. Morozovsky for the stimulating discussion of the considered problems.

\section{References}

1. R. Kretschmer and K. Binder, Surface effects on phase transition in ferroelectrics and dipolar magnets // Phys. Rev. B 20 (3), pp.1065-1076 (1979).

2. Y. G. Wang, W. L. Zhong and P. L. Zhang, Surface and size effects on ferroelectric films with domain structures // Phys. Rev. B 51 (8), pp.5311-5314 (1995).

3. A. M. Bratkovsky and A. P. Levanyuk, Abrupt appearance of the domain pattern and fatigue of thin ferroelectric films // Phys. Rev. Lett. 84 (14), pp.3177-3180 (2000).

4. G. E. Pike, W. L. Warren, D. Dimos, B. A. Tuttle, R. Rames, J. Lee, V. G. Keramidas, J.T.Evans, Voltage offsets in $(\mathrm{Pb}, \mathrm{La})(\mathrm{Zr}$, Ti) $\mathrm{O}_{3}$ thin films // Appl. Phys. Lett. 66 (4) pp.484-486 (1995).

5. G. Suchaneck, T. H. Sander, R. Kohler, G. Gerlach, Investigation of the spatial polarization distribution of sputtered PZT thin films using LIMM // Integrated Ferroelectrics 27, pp.127-136 (1999).

6. Y. G. Wang, W. L. Zhong and P. L .Zhang, Surface effects and size effects on ferroelectrics with a first-order phase transition // Phys. Rev. B 53 (17), pp.11439-11443 (1996).

7. Y. Ishibashi, H. Orihara and D. R. Tilley, Thickness transitions of ferroelectricity in thin films // J. Phys. Soc. Jap. 67 (9), pp.3292-3297 (1998).

8. B. D. Qu, W. L. Zhong and R. H. Prince, Interfacial coupling in ferroelectric superlattices // Phys. Rev. B 55 (17), pp.1121811224 (1997).

9. V. M. Fridkin, Ferroelectrics semiconductors, Consult Bureau, New York (1980).

10. I. S. Gradshteyn and I. M. Ryzhik, Tables of integrals, Physmatgis, Moscow (1963).

11. A. Erdelyi et al., Higher Transcendental Functions, vol. 3, McGraw-Hill Book Company, Inc., New York (1955)

12. M. D. Glinchuk, E. A. Eliseev and V. A. Stephanovich, Size effects of pyroelectric coefficient and dielectric susceptibility in ferroelectric thin films // cond-mat/0103083.

13. W. Y. Shih, W.-H. Shih and I. A. Aksay, Size dependence of the ferroelectric transition of small $\mathrm{BaTiO}_{3}$ particles: Effect of depolarization // Phys. Rev. B 50 (21), pp.15575-15585 (1994). 